\title{
Cultura organizacional y sistemas de gestión de la calidad: una relación clave en la gestión de las instituciones de educación superior ${ }^{*}$
}

\section{Organizational Culture and Quality Management Systems: A Key Relationship in the Management of the Higher Education Institutions}

\section{Juan Javier Vesga R.}

Referencia para citar este artículo: VESGA, J. J. (2013). "Cultura organizacional y sistemas de gestión de la calidad: una relación clave en la gestión de las instituciones de educación superior". En: Revista Guillermo de Ockham 11(2). pp. 89-100.

\section{Resumen}

El presente artículo es una discusión acerca de la importancia de la cultura organizacional en la implementación de sistemas de gestión de la calidad en instituciones de educación superior. Con base en las propuestas de algunos autores, se estudia la necesidad de pensar la cultura de una organización como un componente clave de los planes de implementación de sistemas de gestión de la calidad, a fin de alinear la cultura con las estrategias de gestión ya sea adecuando los sistemas de gestión a la cultura existente o interviniendo la cultura para integrarla con la estrategia de gestión, de acuerdo con la perspectiva de cultura organizacional adoptada por los líderes de las instituciones.
Palabras clave: cultura organizacional, sistemas de gestión de la calidad, gestión de la cultura, instituciones de educación superior.

\section{Abstract}

In this article, the author creates a discussion about of the importance of organizational culture in the implementation of the quality management system in higher education institutions. The author considers, based on the proposals of some authors, the need to study the culture of an organization as a key component of the plans for the implementation of the quality management system to align culture with management strategies, either adapting management

- Fecha de recepción del artículo: 13-04-2013 • Fecha de aceptación: 09-07-2013

JUAN JAVIER VESGA R. Psicólogo, Especialista en Gestión de Talento Humano. Magíster en Psicología y candidato a Doctor en Psicología de la Universidad del Valle. Docente en la Facultad de Psicología de la Universidad de San Buenaventura Cali. Correo electrónicos: jjvesga@ usbcali.edu.co.

* El presente artículo de revisión y reflexión es producto del proyecto de investigación "Caracterización de la cultura organizacional de una Institución de Educación Superior como plataforma para la implementación de un sistema de gestión de la calidad” del Grupo de Investigación "Estéticas Urbanas y Socialidades" de la Facultad de Psicología de la Universidad de San Buenaventura Cali. Fecha de inicio: febrero de 2010. Fecha de finalización: febrero de 2011. 
systems to the existing culture or intervening the culture to align it with the management strategy, depending on the perspective on organizational culture adopted by the leaders of the institutions.

Keywords: organizational culture, quality management systems, culture management, culture management, higher education institutions.

\section{Introducción}

La implementación de sistemas de gestión de la calidad es una de las estrategias de manejo que han venido implementando en la última década los líderes de las instituciones de educación superior (IES) en Colombia, como mecanismo para aumentar su competitividad en el mercado y responder a las exigencias de alta calidad que ha estado impulsando el Gobierno colombiano a partir de la Ley 30 de 1992 y los decretos posteriores que plantean exigencias y retos en materia de alta calidad. De esta manera, diversas instituciones como la Universidad Militar Nueva Granada, la Universidad de San Buenaventura, la Universidad Icesi, la Universidad del Valle y la Universidad Tecnológica de Bolívar, entre otras, han incorporado esta estrategia de gestión (Vesga, 2011).

Sin embargo, independientemente del nivel de desarrollo estratégico que tenga la organización en cuanto al proceso de incorporación de modelos de gestión, ya se trate de sistemas de gestión de la calidad o de cualquier otro modelo, suelen presentarse obstáculos para su implementación originados en comportamientos y hábitos arraigados en las personas que conforman la organización, lo que lleva a la búsqueda de alternativas para la aplicación de técnicas en el manejo de la "resistencia al cambio", acciones estas que terminan siendo en muchas ocasiones tan solo un paliativo para las situaciones "problemáticas" presentadas. Al respecto, Méndez (2006) manifiesta que "los resultados fallidos de la aplicación de tecnologías de gestión puede explicarse en el contexto de la cultura organizacional, [ya que] se aplican y se adaptan modelos de gestión a personas que comparten una cultura organizacional contraria a los requerimientos para su aplicación" (p. 16). En tal sentido, es necesario comprender, entonces, que la cultura de una organización es factor clave para el éxito o el fracaso en la implementación de los sistemas de gestión; o como lo afirma Serna (2008), "la cultura corporativa es, por tanto, una de las mayores fortalezas de una organización si coincide con sus estrategias. Pero si es no ocurre, será una de sus principales debilidades" (p. 133).

"La puesta en marcha de las estrategias gerenciales deberá considerar los elementos culturales que las facilitan u obstaculizan" (Serna, 2008, p. 133), lo que allanará el camino para la implementación del modelo de gestión adoptado. Esto implica que una parte prioritaria e inicial del plan deberá comprender el análisis de la cultura organizacional y su relación con los sistemas de gestión en la organización en particular. En este sentido, este autor afirma que "uno de los mayores errores gerenciales está en separar la cultura organizacional de las estrategias corporativas. Es importante que, cuando estas se definan, incluyan programas y acciones que coincidan o, si se requiere, modifiquen los elementos culturales que impidan o faciliten la puesta en marcha de las estrategias" (Serna, 2008, p. 134).

En este artículo se presentan algunas reflexiones fundamentales acerca de las relaciones entre cultura organizacional y sistemas de gestión de la calidad en el marco de la dirección de las IES, con el propósito de ofrecer un aporte teórico con orientación empírica a quienes de una u otra forma son gestores o apoyan procesos de cambio en el momento de implementar modelos de gestión.

\section{La calidad en las IES}

La evolución de la especie humana ha estado marcada desde siempre por el deseo de un mejoramiento continuo, lo que ha conducido al desarrollo cada vez más complejo de estrategias para la solución de sus necesidades tanto individuales como colectivas, desde su habitar en las cavernas hasta los viajes espaciales de la actualidad. Esto ha conllevado la búsqueda de desarrollos clave de tecnologías y saberes que respondan con eficiencia y eficacia a los problemas planteados en las sociedades de todos los tiempos.

Esta indagación continua por hacer las cosas cada vez mejor, se encuentra orientada hacia la calidad en el quehacer cotidiano, muy particularmente en la acción de las organizaciones empresariales. Sin embargo, es a partir de la finalización 
de la Segunda Guerra mundial que toma auge el movimiento de la calidad como estrategia de las organizaciones para pervivir en los mercados cada vez más competidos y en las dinámicas de un mundo globalizado.

Como punto de partida de la reflexión sobre calidad, este concepto se define como "propiedad o conjunto de propiedades inherentes a algo, que permiten juzgar su valor" (Real Academia Española, 2006). En este sentido, la calidad se refiere fundamentalmente a la relación que puede existir en un momento dado entre las características que definen o describen la naturaleza de algo y el valor de uso que posee para una persona o para una colectividad.

Discernir sobre la calidad no es un asunto fácil, pues como bien lo expresan Camisón, Cruz, y González (2007) "existe sobre el concepto de calidad un auténtico diluvio de definiciones redundantes, que contribuyen poco a clarificar su significado actual" (p. 145). En otro sentido y desde una perspectiva más empresarial, estos mismos autores manifiestan que la gran cantidad de términos asociados al concepto de calidad puede llevar al desconcierto entre los directivos de empresas, trayendo como consecuencia la falta de rigor y de acuerdos en su significado (Camisón, Cruz, y González, 2007). Si bien es cierto que la calidad ha sido definida desde diversas perspectivas, Moreno, Peris, y González (2001) plantean cuatro categorías dentro de las cuales, pueden agruparse la mayoría de las definiciones de la calidad.

La primera se refiere a "la calidad entendida como conformidad con unas especificaciones" (p.8). Desde esta perspectiva, la calidad corresponde al cumplimiento de unos requerimientos previamente establecidos o a unos estándares definidos por alguna autoridad en la materia. La segunda categoría corresponde a la perspectiva de "la calidad como satisfacción de las expectativas del cliente" (p.9), lo cual lleva a que la calidad esté determinada por el cliente en función de su nivel de satisfacción y no necesariamente por alguna autoridad o estándar establecido. En este caso, un mismo producto o servicio puede tener diferentes niveles de calidad de acuerdo con la percepción de cada cliente en función de la satisfacción de sus deseos o necesidades. La tercera corresponde a "la calidad como valor en relación con el precio" (p.11), lo cual lleva a que la calidad esté definida por la relación entre la satisfacción recibida o el uso posible y el precio pagado por el producto o servicio. Finalmente está el grupo de las teorías que presentan "la calidad como excelencia" (p.12), lo que implica no solo las características finales del producto o la satisfacción percibida por el cliente, sino también las condiciones del proceso de elaboración del producto o prestación del servicio (Moreno, Peris, y González, 2001).

En cuanto al concepto de calidad en la educación superior, existe una gran complejidad y multiplicidad en su uso y en la definición del concepto, ya que "en el ámbito educativo [...] se tienen diferentes conceptos, apreciaciones y visiones de la expresión calidad educativa" (Giraldo, Abad, y Díaz, 2003, p. 156). Para estos autores, "la falta de una teoría sobre la calidad y las dificultades para poder llegar a un consenso entre las distintas audiencias implicadas impiden que se pueda establecer una definición de este concepto que sea aceptada de forma unánime" (p. 156).

La noción de calidad en la educación tiene muchas aristas. Puede estar relacionada con las metodologías de enseñanza que permitan eficacia en los aprendizajes, con los contenidos temáticos de los programas académicos y su pertinencia en relación con las necesidades del contexto, con el desarrollo de competencias de los estudiantes y su repercusión en el medio externo, con la pertinencia de los saberes aportados en la formación de los futuros profesionales y con las condiciones de las instalaciones físicas y equipos de apoyo metodológico, entre otros; y eso por hablar tan solo de una de las funciones sustantivas como es la de docencia, ya que si se analiza la cuestión con base en las funciones de investigación y extensión, el asunto se vuelve mucho más complejo.

Para Nicoletti (2008) "la calidad de la educación no deja de ser un concepto normativo y multifactorial, donde intervienen dimensiones de la filosofía, la pedagogía, la cultura, la sociedad y la economía” (p. 76). Para este autor, resulta fundamental definir la perspectiva desde la cual mirar la calidad, por lo cual y siguiendo las ideas de Muñoz Izquierdo (2003), Nicoletti manifiesta que según una mirada pedagógica la calidad estaría en función del logro de los objetivos de los currículos, asociando calidad al concepto de eficacia. Desde la 
perspectiva de la cultura, la calidad en la educación estaría en función de su respuesta a las condiciones, posibilidades y aspiraciones de los grupos sociales y en asocio al concepto de pertinencia. Desde el punto de vista social, una educación de calidad sería aquella que puede ser distribuida equitativamente entre diversos sectores de la sociedad; y desde el punto de vista económico, la calidad en la educación estaría en función de la manera como se aprovechen los recursos utilizados para impartirla y en asocio al concepto de eficiencia (Nicoletti, 2008).

Una forma de acercarse al concepto de calidad en la educación superior son los lineamientos establecidos por los sistemas de evaluación de los organismos de acreditación de la calidad creados por los gobiernos en diferentes países, los cuales proponen definiciones de calidad a partir del establecimiento de estándares reguladores. En Colombia, el Consejo Nacional de Acreditación (2012), al definir el concepto de calidad expresa lo siguiente:

El concepto de calidad aplicado al bien público de la educación superior hace referencia a la síntesis de características que permiten reconocer un programa académico específico o una institución de determinado tipo y hacer un juicio sobre la distancia relativa entre el modo como en esa institución o en ese programa académico se presta dicho servicio y el óptimo que corresponde a su naturaleza.

Para aproximarse a ese óptimo, el Consejo Nacional de Acreditación ha definido un conjunto de características generales a partir de las cuales se emiten los juicios sobre la calidad de instituciones y programas académicos, pero la determinación más específica y el peso relativo de esas características estarán, en buena parte, condicionados a la naturaleza de la institución y a la del programa académico en cuestión. En este contexto, un programa académico tiene calidad en la medida en que haga efectivo su proyecto educativo, en la medida en que se aproxime al ideal que le corresponde, tanto en relación con sus aspectos universales, como con el tipo de institución al que pertenece y con el proyecto específico en que se enmarca y del cual constituye una realización. La calidad, así entendida, supone el esfuerzo continuo de las instituciones por cumplir en forma responsable con las exigencias propias de cada una de sus funciones (p. 14).

En este orden de ideas y de acuerdo con la clasificación propuesta por Moreno, Peris y González
(2001), esta definición se ubica en la perspectiva de la calidad entendida como conformidad con unas especificaciones, las cuales en este caso son determinadas por un organismo gubernamental regulador. Sin embargo -manifiestan estos autores- "la aplicación de este concepto se vuelve más problemática cuando sus defensores (Juran, Deming y Crosby, entre otros), insisten en que las especificaciones deben ser definidas en función de los requerimientos del cliente" (pp. 8,9), enfoque manejado por organismos internacionales como la ISO (International Organization for Standardization) en la implementación de sistemas de gestión de la calidad. En este sentido, la norma técnica colombiana NTC-ISO 9000 (Icontec, 2009) define la calidad como "el grado en el que un conjunto de características inherentes cumple con los requisitos" (p. 9) y el requisito es definido como la "necesidad o expectativa establecida" (p. 9).

Dado que las instituciones de educación superior están reguladas por organismos gubernamentales los cuales establecen un conjunto de estándares que deben ser cumplidos como condición de calidad, la perspectiva de la conformidad con las especificaciones es pertinente a este tipo de organizaciones. Sin embargo, es recomendable complementar esta visión con una perspectiva a partir de las necesidades, expectativas y satisfacción de los usuarios o clientes, ya sean estos estudiantes en formación, organizaciones que los contratan una vez sean profesionales o la sociedad en general que se ve afectada por la acción de la educación superior.

\section{Gestión de la calidad}

Independientemente de la perspectiva que se adopte para definir la calidad o de que se decida tenerlas en cuenta a todas, la calidad en las organizaciones debe ser gestionada pues esta no se logra por sí misma sino que requiere de procesos de gestión encaminados a lograr los niveles de calidad establecidos ya sea por la propia organización, las partes interesadas o por alguna autoridad nacional $\mathrm{o}$ internacional reguladora.

La expresión "gestión de la calidad" es definida por Camisón, Cruz y González (2007) como "un sistema que relaciona un conjunto de variables relevantes para la puesta en práctica de una serie 
de principios, prácticas y técnicas para la mejora de la calidad" (p. 211). En este sentido, gestionar la calidad implica diseñar un sistema que ponga en relación diversos componentes y aspectos de la organización relativos a los resultados asociados a la concepción de calidad. Por su parte la NTC-ISO $9000^{1}$ (ICONTEC, 2009) define el sistema de gestión de la calidad (SGC) como "aquella parte del sistema de gestión de la organización enfocada en el logro de resultados, en relación con los objetivos de la calidad, para satisfacer las necesidades, expectativas y requisitos de las partes interesadas, según corresponda” (p. 8).

La gestión de la calidad ha evolucionado en el tiempo especialmente a partir de la Revolución Industrial. Varios autores (Moreno, Peris y González, 2001; Camisón, Cruz y González, 2007) coinciden en señalar una serie de etapas en los enfoques de gestión de la calidad.

La primera etapa comprende desde finales del siglo XIX hasta 1930 y se caracteriza por una gestión orientada hacia la inspección final del producto terminado y separada del proceso de producción. En 1931 se da la segunda etapa cuando un equipo de ingenieros de la Bell Telephone Laboratories dirigido por Walter Shewhart, desarrolló una serie de técnicas para monitorear y evaluar la calidad durante el proceso de producción mediante la introducción de herramientas estadísticas (especialmente teorías de probabilidad) que permitían identificar variaciones en los productos. Una tercera etapa denominada de aseguramiento de la calidad, surge hacia los años sesenta, época en la que se da un cambio en la gestión cuando se pasa de la detección de los errores a su prevención lo cual llevó a observar el proceso completo, desde la selección de los materiales hasta su control permanente a fin de "asegurar" la calidad desde el principio. En esta etapa son especialmente importantes los aportes de Edward Deming (1900-1993) (Moreno, Peris, y González, 2001).

Con el interés cada vez más centrado en la calidad y la gestión como una forma de diseñar, concebir y gobernar las organizaciones de trabajo, surge en 1947 en Ginebra, Suiza, la Organización Internacional de normalización (ISO por sus siglas en inglés: International Standardization Organization), organismo dependiente de la ONU cuyo propósito es instituir estándares internacionales de calidad. Este organismo ha publicado una serie de normas internacionales que regulan la calidad en diversos aspectos, como la familia ISO 9000 referida a la gestión y aseguramiento de la calidad; ISO 14000 relacionada con la gestión ambiental, y la ISO 22.000 que atañe al sistema de gestión de la inocuidad de los alimentos, entre otras.

La implementación de sistemas de gestión de la calidad basados en las normas ISO es una estrategia de gran aceptación en el mundo por parte de organizaciones de diverso tipo. Algunos estudios acerca del efecto que la implementación de sistemas de gestión de la calidad tiene en el desempeño de las organizaciones, muestran que en general las empresas mejoran su desempeño al implantar este tipo de estrategias de gestión. Ejemplo de ello son los resultados encontrados por Nava y Rivas (2008), quienes llevaron a cabo una investigación para identificar si el desempeño de las empresas mexicanas había mejorado como resultado de la implementación de sistemas de gestión de la calidad. La investigación se llevó a cabo con 96 organizaciones mexicanas y se observó que el desempeño de una organización sí mejora cuando se implanta un programa ISO 9000, siempre y cuando se dé una motivación activa; es decir, cuando la adopción de la estrategia no es impuesta por un agente externo, sino que se instituye como consecuencia de un proceso participativo (Nava y Rivas, 2008).

En Colombia, el Icontec ${ }^{2}$ encargó al Centro Nacional de Consultoría una investigación para determinar los efectos de la implementación de sistemas de gestión de la calidad en las empresas colombianas certificadas por este y otros organismos de certificación durante el período 2000-2005. Para efectos del estudio, se entrevistaron a 563 empresarios de los cerca de cuatro mil que certificaron sus compañías en ese periodo. En esta investigación, el $92 \%$ de ellos denotó un incremento de la satisfacción del cliente con la implementación del SGC y la obtención de la certificación. Para el $23 \%$ la exigencia de la certificación ISO 9001 se la hace más del $71 \%$ de sus clientes, lo que

1. Norma técnica colombiana-ISO 9000

2. Instituto Colombiano de Normas Técnicas. Es un organismo privado que trabaja para fomentar la normalización, la certificación, la metrología y la gestión de la calidad en Colombia. 
evidencia la importancia que la certificación de los sistemas de gestión de la calidad tiene para acceder a los mercados. En cuanto a las ventas, a partir del primer año de certificación estas tienen una tendencia de crecimiento de alrededor del 5,5\% y el crecimiento, dos años después de la obtención de la certificación, es del $12 \%$. Finalmente, respecto al desempeño en general de la organización, el $95 \%$ de los empresarios expresó que la certificación ha sido positiva para sus empresas, lo cual es congruente con el incremento de satisfacción del cliente expresado por los empresarios (Icontec, 2006).

La implementación en la educación superior de sistemas de gestión de la calidad basados en las normas ISO, no implica la "estandarización" de productos sino la normalización de procesos. Al respecto, Nicoletti (2008) afirma que

la estandarización de un sistema de calidad, no implica que los bienes, servicios o formación de los alumnos tengan que ser iguales en todo el mundo; la normalización de los sistemas y procesos apela al diseño particular de lo que la organización desea lograr, a la documentación de cómo se hacen las cosas y a la verificación de cómo se hicieron (p. 79).

De acuerdo con este autor, los beneficios que aporta a las instituciones educativas la implementación de un sistema de gestión de la calidad son, entre otros, "una mejor administración de los procesos sutantivos; un mejor control de las áreas clave, una medición de un proceso de mejora; [y] un mayor involucramiento del personal en sus actividades diarias" (p. 83).

\section{Cultura organizacional}

El concepto de "cultura organizacional" ha sido estudiado por una gran variedad de autores $\mathrm{y}$ aunque hay similitudes entre las diferentes concepciones, prevalece una ausencia de consenso sobre su definición. Para Gómez, Sánchez y del Almo (2005) los debates alrededor de esta noción hunden sus raíces en las diferencias existentes entre los paradigmas científicos que los investigadores asumen al abordar el estudio de este fenómeno, lo que conlleva posturas divergentes no solo en cuanto a su conceptualización sino en relación con las metodologías utilizadas para investigarlo.
Con el propósito de sintetizar las diversas alternativas de definición propuestas, García (2009) agrupa las distintas conceptualizaciones sobre cultura organizacional agruparse en dos grandes perspectivas: las emergentes y las racionales.

Desde las perspectivas emergentes, la cultura es vista como un conjunto de significados compartidos y como una construcción subjetiva de los miembros de la organización. En tal sentido, la cultura no sería una realidad externa a los sujetos sino producto de la subjetivación que estos hacen de la realidad que experimentan respecto de los entornos en que se desenvuelven (García, 2009).

Hatch (1997) se refiere a esta perspectiva como la teoría simbólico-interpretativa de la cultura organizacional fundamentada en el supuesto de que las culturas son realidades construidas socialmente. En tal sentido, los seres humanos pueden participar de los aspectos de la vida organizacional socialmente construida debido a que hacen uso de símbolos y los interpretan, los cuales crean y mantienen la cultura organizacional (Hatch, 1997). De acuerdo con esta autora, dos aspectos son claves al momento de definir y comprender el concepto de cultura organizacional desde esta perspectiva: los símbolos y su interpretación: "Un símbolo es cualquier cosa que represente una asociación consciente o inconsciente con algún concepto o significado más amplio. Así, un símbolo consiste de una forma tangible y de un significado (o significados) más amplio con el que la misma está asociada (p. 219).

Desde las perspectivas emergentes o simbólicointerpretativas, la construcción de significados compartidos a partir de la creación de símbolos es clave para la formación y consolidación de culturas organizacionales. No obstante, los símbolos son de sí ambiguos, por lo que los miembros de una organización podrían darles diferentes significados (Hatch, 1997), razón por la cual en las organizaciones se establecen mecanismos de influencia para la construcción de significados socialmente compartidos, aunque es recomendable que sus líderes hagan esfuerzos por conocer y comprender los significados que de manera autónoma se construyen alrededor de ciertos elementos que llegan a tornarse simbólicos o representativos de su cultura. 
En este punto se hace necesario hacer claridad sobre algunos matices clave para la comprensión de lo que significa una perspectiva emergente o simbólico-interpretativa referidos a la diferencia entre las miradas constructivistas y las construccionistas.

Aunque ambas se refieren a una realidad no externa al individuo sino a su construcción a partir de la formación de significados, el constructivismo -que proviene de la psicología del desarrollo- se centra más en los procesos cognitivos individuales. De otra parte, el construccionismo -que proviene de la psicología social- según Gergen (1996) se aparta del constructivismo en que lo fundamental no son los procesos cognitivos perceptuales en un nivel individual sino los procesos de interacción social. "El construccionismo busca dar cuenta de las construcciones que los individuos elaboran colectivamente, mientras que el constructivismo busca dar cuenta de la construcción de las estructuras cognitivas que el individuo elabora en el transcurso de su desarrollo" (Arendt, 2003, p. 5). En tal sentido, definir la cultura como una construcción de los individuos a partir de significados construidos, implica tener claridad acerca de si tal definición se sustenta en una mirada cognitivo-individual o en una perspectiva de construcción social.

Las perspectivas emergentes no hablan de una gestión de la cultura propiamente dicha, sino de la intervención orientada en sentido inverso; esto es, diseñar o adaptar los sistemas de gestión a la cultura existente en la organización a partir de su comprensión para que de esta manera las estrategias estén en sintonía con la cultura existente en la organización.

De otra parte y de acuerdo con la propuesta de García (2009), se encuentran las perspectivas racionales a partir de las cuales se entiende la cultura como una realidad externa a los individuos que puede ser conocida, intervenida y gestionada en procura de una mayor efectividad en el desempeño organizacional.

El paradigma racional contempla a la organización como un producto de decisiones racionales basadas en la aplicación de conocimientos científicos tales como la Psicología, que busca alcanzar el bienestar y la eficiencia de la organización [...]. Desde esta perspectiva, la cultura se considera como una dimensión organizacional que puede ser intervenida a través de diversas estrategias, con el fin de lograr la adaptación de la organización a los cambios del mercado. La cultura es concebida como una variable que puede ser transformada a través de técnicas, con el fin de alinear los comportamientos de las personas con los objetivos corporativos (García, 2009, p. 279).

Esta perspectiva predomina en el lenguaje administrativo y en las teorías gerenciales. Los discursos gerenciales sobre el cambio organizacional, han estado fuertemente asociados a las técnicas de intervención para la transformación de las culturas organizacionales como factor clave para lograr un alineamiento del desempeño del personal en función de las estrategias corporativas. En tal sentido, el estudio de la cultura no solo se orienta a su comprensión teórica o conceptual, sino que sirve como mecanismo o técnica para la intervención del sistema organizacional en alineación con su estrategia (García, 2009). De esta manera, es posible entender que se puede dar una "gestión de la cultura" como estrategia gerencial para el logro de los objetivos organizacionales. Así, los componentes de la cultura (aspectos físicos de la organización, patrones de comunicación e interacción social, rituales, etc.) pueden ser intencionalmente "administrados" contingentemente con los planes estratégicos de la organización.

Finalmente, ¿qué se entiende por cultura organizacional? Para Fernández (2002) esta se define como

Un sistema de valores, nacido, desarrollado y consolidado en un entorno determinado, que se expresa y transmite a través de una red de símbolos (ritos, mitos, pautas de conducta, artefactos etc.) históricamente determinado y determinante, y que al ser más o menos compartido por los subgrupos de la organización presta por una lado la necesaria integración y por otro da lugar a las diferentes subculturas, entre las que con frecuencia se origina el conflicto más o menos manifiesto por el acceso al control tanto ideológico como de los medios con que cuenta la organización para la consecución de sus fines (p. 11).

Este autor clasifica de los componentes de la cultura organizacional en formales o expresivos y nucleares, los cuales se muestran en la siguiente Tabla: 
Tabla 1

Componentes de la cultura organizacional propuestos por Fernández, J.M. (2002)

\begin{tabular}{|c|c|c|c|}
\hline \multirow{5}{*}{ 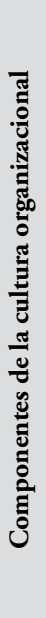 } & \multirow{4}{*}{$\begin{array}{c}\text { Formales o } \\
\text { expresivos }\end{array}$} & Artefactos materiales & $\begin{array}{l}\text { Tecnología } \\
\text { Distribución del espacio } \\
\text { Decoración de edificios } \\
\text { Forma de vestir }\end{array}$ \\
\hline & & Formas lingüísticas & $\begin{array}{l}\text { Lenguaje } \\
\text { Mitos, leyendas e historias }\end{array}$ \\
\hline & & Personajes & $\begin{array}{l}\text { Fundadores } \\
\text { Héroes } \\
\text { Líderes }\end{array}$ \\
\hline & & Ritos, rituales & $\begin{array}{l}\text { Ritos sociales y de comunicación } \\
\text { Ritos de trabajo } \\
\text { Rituales administrativos } \\
\text { Rituales de reconocimiento }\end{array}$ \\
\hline & Nucleares & $\begin{array}{l}\text { De contenido } \\
\text { y significado }\end{array}$ & $\begin{array}{l}\text { Valores } \\
\text { Creencias } \\
\text { Presunciones básicas }\end{array}$ \\
\hline
\end{tabular}

Fuente: Elaboración propia a partir de Fernández J.M. (2002)

Los elementos formales o expresivos son "la parte visible de la cultura, la parte más accesible de la misma, tanto para el observador externo como para la conciencia de los sujetos que forman la organización" (Fernández, 2002, p. 23). De otra parte, están los elementos nucleares, que son aquellos que constituyen "el significado o contenido de la cultura al que hacen referencia los elementos formales [...]. Constituyen el núcleo de la cultura, la referencia última de los distintos elementos culturales de una organización" (Fernández, 2002, pp. 32, 33).

Por su parte para Méndez (2006) "la cultura organizacional es la conciencia colectiva que se expresa en el sistema de significados compartidos por los miembros de la organización que los identifica y diferencia de otros institucionalizando y estandarizando sus conductas sociales" (p. 91). Este autor propone cuatro dimensiones implícitas en la organización que permiten identificar rasgos características de la cultura de una organización: el concepto del líder sobre el hombre, la estructura de la organización, el sistema cultural y el clima de la organización.

En cuanto al primer aspecto, el autor manifiesta que "la idea sobre la naturaleza de la conducta humana lleva al fundador o líder a construir un concepto o idea ${ }^{3}$ sobre los empleados que deter- mina el marco para las relaciones sociales en la organización" (p. 96). En relación con el segundo aspecto, afirma que la estructura organizacional "asigna claramente responsabilidades y determina parámetros de comportamiento laboral [...]. Identifica la dinámica sobre la que opera la organización en términos de poder, toma de decisiones a partir de la jerarquización, autoridad, centralización y coordinación" (p. 106). El tercer aspecto constituye lo que de manera general ha sido incorporado en el concepto de cultura organizacional. Para este autor, está conformado por los mitos, la ideología, los valores, los ritos, las creencias, los hábitos y las normas e historias de la organización.

En relación con el cuarto y último aspecto, el autor del presente artículo no está de acuerdo en incluirlo como parte de los componentes constitutivos de la cultura organizacional, pues ha quedado claro en la literatura de la psicología organizacional que aunque estos dos conceptos tienen elementos que los relacionan albergan diferencias claves.

Algunas de las diferencias fundamentales se presentan a continuación. Generalmente, los estudios de clima organizacional están dirigidos a "medir" el clima, lo que implica métodos cuantitativos y el uso de encuestas para la obtención de información, mientras que la cultura no se puede medir sino caracterizar o describir, lo cual conlleva 
el uso de métodos cualitativos a partir de observaciones, entrevistas y otros métodos etnográficos. El clima puede ser tan cambiante como el clima atmosférico, metáfora en la cual se fundamenta la expresión y puede ser modificada rápidamente con una intervención, mientras que la cultura requiere un largo periodo si se quieren modificar algunos de sus aspectos ${ }^{4}$.

\section{Cultura organizacional y sistemas de gestión de la calidad}

Hablar de cultura organizacional y su relación con un sistema de gestión implica elegir una perspectiva teórica desde la cual puedan encontrarse los referentes conceptuales en los cuales se basa la construcción de un plan para articular la cultura con la estrategia emanada del sistema de gestión adoptado.

Cualquiera que sea la perspectiva teórica adoptada sobre la concepción de cultura organizacional -desde las perspectivas racionales o desde las emergentes-, es necesario tener en cuenta que para que se conduzcan con mayor probabilidad de éxito los sistemas de gestión en las organizaciones se debe partir siempre de la caracterización o análisis de la cultura; esto es, hacer un estudio para conocer y comprender los aspectos que la definen. Acto seguido, se efectúa una comparación de estos resultados con las características de los sistemas de gestión con el propósito de identificar el grado en que los factores de la cultura y los sistemas de gestión son coherentes entre sí.

Si se ha optado por una perspectiva desde las teorías emergentes y se identifican características de los sistemas de gestión que son improcedentes con la cultura de la organización, se hace necesario adaptar los sistemas a la cultura. Si por el contrario, se opta por una perspectiva a partir de las teorías racionales y al hacer el estudio de la cultura organizacional se encuentra aspectos que dificultarían la puesta en marcha de los sistemas de gestión, se debe intervenir la cultura para modificar aquellos que se requieran antes de poner en marcha tales sistemas de gestión. En este sentido, afirma Méndez (2006) que "la cultura organizacional puede gestionarse en el sentido de que puede ser cambiada mediante intervenciones a través del desarrollo organizacional, entendiendo que este es un proceso de cambio planeado" (p. 22).

Concebir que la cultura organizacional pueda ser gestionada, implica definirla como una variable interna de la organización. "Desde esta concepción se asume que las organizaciones poseen una cultura, es decir, la cultura es una variable que puede ser modificada a través de diversas estrategias gerenciales" (García, 2006, p. 165) lo cual lleva a pensar que al implementar un sistema de gestión, la cultura puede ser intervenida para alinearla con la estrategia mediante el diseńo de planes dirigidos a lograr un cambio cultural.

El cambio cultural desde esta perspectiva, se concibe como un proceso que se da de manera planeada y unidireccional, es decir, los valores y políticas institucionales determinados por la dirección se difunden por toda la organización, con la intención de que éstos sean internalizados por las personas y de esta forma construir compromiso e identidad organizacional por parte de los empleados (García, 2006, p. 166).

¿Qué elementos de la cultura deben ser intervenidos en un proceso de cambio cultural? Todo depende de las relaciones existentes entre las características de los sistemas de gestión y los aspectos de la cultura que facilitan o dificultan su puesta en marcha. Por ejemplo, si uno de los aspectos que describen una cultura es la dificultad de los empleados para trabajar en equipo entre las diferentes unidades y la organización, se propone la puesta en marcha de un sistema de gestión de la calidad basado en la norma ISO 9001, el cual implica concebir y actuar en la organización desde la perspectiva de procesos transversales a las distintas unidades; uno de los requisitos indispensables para el éxito de la estrategia es el trabajo en equipo entre las unidades. En este caso, una parte del plan de gestión de la cultura organizacional debe dirigirse a superar esta debilidad generando estrategias encaminadas a desarrollar un aprendizaje organizacional orientado al trabajo en equipo y a la coordinación entre las distintas unidades organizacionales. 
Camisón, Cruz y González (2007) proponen dos elementos fundamentales que permiten gestionar una cultura organizacional: las políticas de recursos humanos y el liderazgo. Estos autores manifiestan que si, por ejemplo, "la dirección tiene la voluntad de gestionar la cultura organizativa de manera deliberada y proactiva, articulará procesos de selección en los que tendrá gran relevancia la compatibilidad entre los valores individuales de los candidatos y los valores de la organización" (p. 1197). Por su parte, Olea (1995), afirma que algunos factores que resultan importantes en el cambio de la cultura hacia la calidad son: a) crear conciencia en los trabajadores sobre los principios de la calidad; b) obtener el compromiso de todos los miembros de la organización; c) lograr que los miembros de la organización se identifiquen con el sistema de gestión; $y$ d) educación y entrenamiento en sistemas de gestión de la calidad.

De otra parte, Méndez (2006) propone tres momentos en el proceso de cambio y transformación de la cultura. El primero se relaciona con la descripción de la cultura organizacional como insumo para el cambio, "para diseñar estrategias coherentes y factibles de ejecutar dentro de los procesos de cambio organizacional" (Méndez, 2006, p. 22). Al proponer la descripción de la cultura como primer paso para su gestión, este autor pone gran énfasis en el concepto de descripción y no de medición. En este sentido, afirma con vehemencia que "la investigación que describe la cultura de la organización no es un diagnóstico. [...] La cultura organizacional no se mide, se describe" (p. 20). Para este autor, el primer momento comprende la identificación de la forma como las variables de la cultura "afectan la conciencia colectiva de sus miembros y sobre la cual han de diseñarse estrategias de intervención, mediante la capacitación y formación que modifiquen los comportamientos colectivos garantizando hacia el futuro la eficiencia en la gestión" (p. 196).

El segundo momento comprende la identificación de las acciones de intervención, las cuales deben corresponder con las situaciones encontradas en la descripción de la cultura y con los objetivos estratégicos definidos en el marco de la implementación de los sistemas de gestión. En este sentido, la alta dirección debe definir los "rasgos ideales" de la cultura que deben ser desarrollados como producto de la intervención, lo que trae como consecuencia que las acciones propuestas son específicas para cada organización según los aspectos encontrados en la descripción de la cultura (Méndez, 2006).

El tercer momento propuesto por Méndez abarca la consolidación de los rasgos característicos de la cultura organizacional como producto de la intervención. Es preciso después de algún tiempo, hacer un nuevo estudio con el propósito de identificar el efecto de las intervenciones en la modificación de conductas o percepciones asociadas a la cultura organizacional (Méndez, 2006). Esto significa que la gestión orientada a alinear la cultura con la estrategia no es un momento que se da de una vez y por siempre en cualquier organización, sino que se debe concebir como un proceso permanente que exige revisiones periódicas, idas y venidas, aciertos y desaciertos, para replantear permanentemente lo que se está haciendo en materia de cultura organizacional. De la misma manera, se requiere tener presente que las estrategias y los sistemas de gestión cambian con el tiempo a fin de responder a las demandas del entorno, por lo que se hace necesario revisar periódicamente su relación con la cultura organizacional.

En el caso de la implementación de sistemas de gestión de la calidad basados en la norma ISO 9000, esta define ocho principios fundamentales: enfoque al cliente, liderazgo, participación del personal, enfoque basado en procesos, enfoque de sistema para la gestión, mejora continua, enfoque basado en hechos para la toma de decisión y relaciones mutuamente beneficiosas con el proveedor (Icontec, 2009). Sin embargo, la aplicación de estos principios por sí mismos no determina una gestión con calidad. Es necesario liderar en la organización un conjunto de acciones conducentes al desarrollo de valores y prácticas cotidianas que faciliten el desempeño de alta calidad con convicción, desde la alta gerencia hasta la base operativa de la organización, lo que implica desarrollar una cultura orientada a la calidad. En este orden de ideas, afirma Vesga (2011):

El análisis y comprensión de la cultura organizacional se hace necesario como punto de partida para la implementación de sistemas de gestión de la calidad, ya que como lo plantean Hellriegel y Slocum (2004) siguiendo a Meyer (2001) "la cultura organizacional representa un patrón complejo de creencias, expectativas, ideas, valores, actitudes y conductas compartidas por los miembros de una organización 
(pág.378). En tal sentido, dicho patrón se establece a partir de las prácticas y saberes cotidianos, por lo que se requiere conocerlos en profundidad para identificar hasta dónde las creencias, expectativas, ideas, valores y actitudes constitutivos de la cultura organizacional, favorecen u obstaculizan la implementación de un nuevo sistema de gestión organizacional (p. 13).

Por esta razón, al planear la implementación de un nuevo sistema de gestión se hace necesario identificar las características que describen la cultura organizacional.

La implementación de un sistema de gestión de la calidad implica un cambio de paradigmas en los sujetos que la conforman, en la manera de ver y comprender la organización por parte de los miembros de la misma, pues las prácticas cotidianas en la gestión de la dinámica organizacional son mediadas por los significados construidos por las personas que la conforman y al instaurar un nuevo sistema de gestión, cambian las prácticas y los sistemas de trabajo, lo que implica necesariamente una resignificación de la relación de los sujetos con el trabajo (Vesga, 2011, p. 12).

\section{Conclusión}

Reflexionar, investigar y teorizar sobre la cultura organizacional y su relación con los sistemas de gestión de la calidad en las IES en Colombia, son acciones que cada día toman mayor relevancia debido, por una parte, al incremento que ha tenido la implementación de estos sistemas de gestión en el sector de la educación en la última década $y$, de otra, al correspondiente señalamiento de diversos autores sobre la importancia de la cultura organizacional en relación con el éxito o fracaso en la implementación de estos modelos de gestión.

La implementación de sistemas de gestión de la calidad resulta de particular importancia para las IES, toda vez que ello constituye un método que permite desarrollar las condiciones óptimas de calidad, en respuesta a las demandas de formación integral de nuevos profesionales que aporten importantes soluciones a las problemáticas que enfrentan las sociedades actuales y cumplir con las condiciones exigidas por organismos nacionales e internacionales reguladores de las características identificadoras de calidad en instituciones de educación superior, como es el caso en Colombia del
Consejo Nacional de Acreditación (CNA), el cual propone una serie de indicadores que deben cumplirse para otorgar la acreditación de alta calidad. Igualmente, la gestión por procesos-componente clave de los sistemas de gestión de la calidad-, se constituye en un elemento articulador de la estructura organizacional y en una importante alternativa de complemento a la tradicional forma de diseñar las organizaciones como estructuras funcionales representadas en los organigramas, dando paso así a la correspondiente representación en el mapa de procesos.

Sin embargo, aunque las características de un sistema de gestión de la calidad están definidas internacionalmente por las normas ISO, cada institución posee unas condiciones únicas que implican que este sistema de gestión debe adaptarse de acuerdo con tales condiciones, particularmente en lo referido a su cultura organizacional. Tal como lo ha afirmado (Méndez, 2006), "se aplican tecnologías de gestión que involucran a las personas de la organización [...] [razón por la cual] primero que todo hay que conocer al hombre y la cultura de la empresa” (p. 31). De acuerdo con este mismo autor, "el conocimiento de la cultura que caracteriza a una organización se logra mediante su 'descripción”" (p. 31), por ello, el autor de este artículo propone la caracterización de la cultura organizacional a partir de su descripción, como uno de los elementos clave para la incorporación de sistemas de gestión de la calidad en instituciones de educación superior, aspecto que también se aplica a todo tipo de organizaciones.

Ya sea que se busque modificar aspectos de la cultura para que el sistema funcione o se adapten los sistemas de gestión a la cultura existente, el reconocer la importancia de la relación entre la cultura organizacional y las dinámicas de los sistemas de gestión puede ahorrar costosos procesos adaptativos en todo tipo de organizaciones.

Un sistema de gestión no es una "receta" que se toma de un "vademécum de herramientas gerenciales" para aplicarla por igual a todas las organizaciones. La cultura de una organización constituye, quizá, el aspecto más representativo de su particularidad y de su identidad, así como de su individualidad, razón por la cual esta debe ser tenida en cuenta al implementar cualquier sistema de gestión. 


\section{Bibliografía}

- ARENDT, R. J. (2003). Construtivismo ou construcionismo? Contribuiçôes deste debate para a Psicologia Social. En: Estudos de Psicologia, 8(1). pp. 5-13.

- CAMISÓN, C., Cruz, S., y González, T. (2007). Gestión de la calidad: conceptos, enfoques, modelos y sistemas. Madrid: Pearson.

- DENISON, D. (1996). What is the difference between organizational culture and organizational climate? A native's point of view on a decade of paradigm wars. In Academy of Management Review, 21(3). pp. 619-654.

- FERNÁNDEZ, J. M. (2002). Cultura de la organización y centro educativo. Madrid: Universidad Complutense de Madrid.

- GARCÍA, C. M. (2006). Una aproximación al concepto de cultura organizacional. En: Universitas Psychologica 5(1). pp. 163-174.

- GARCÍA, C. M. (2009). La cultura organizacional como una tecnología de control gerencial. In: M. C. Aguilar, y E. Rentería. Psicología del trabajo y las organizaciones. Reflexiones y experiencias de investigación. Bogotá, D.C.: Universidad Santo Tomás.

- GERGEN, K. (1996). Realidades y relaciones: aproximaciones a la construcción social. (F. Meller, Trad.) Buenos Aires: Paidós.

- GIRALDO, U., Abad, D. y Díaz, E. (2003). Bases para una política de calidad de la educación superior en Colombia. En: C. N. Acreditación, Educación superior, calidad y acreditación. Bogotá D.C.: CNA.

- GÓMEZ, A. M., Sánchez, J. C. y del Almo, E. A. (2005). Cultura organizacional. En: F. J. Palací, Psicología de la organización. Madrid: Pearson Prentice Hall.

- HATCH, M. J. (1997). Organization Theory. New York: Oxford.

- ICONTEC (2006). Impacto de la certificación de sistemas de gestión de la calidad en las empresas colombianas. Bogotá D.C.: ICONTEC.

- ICONTEC (2009). Normas fundamentales sobre gestión de la calidad y documentos de orientación para su aplicación. Bogotá D.C.: ICONTEC.

- MÉNDEZ, C. E. (2006). Transformación cultural en las organizaciones. Un modelo para la gestión del cambio. Bogotá D.C.: Limusa.

- MORENO, M. D., Peris, F. y González, T. (2001). Gestión de la Calidady Diseño de Organizaciones. Madrid: Prentice Hall.

- NAVA, V. M. y Rivas, L. A. (2008). Desempeño de las organizaciones mexicanas certificadas en la norma ISO 9001:2000. En: Estudios gerenciales, 24 (108). pp.107-128.

- NICOLETTI, J. A. (2008). Adecuación y aplicación de las normas de calidad ISO 9000:2000 en el campo educativo. En: Horizontes educacionales, 3 (2). pp. 75-86.

- OLEA, B. (1995). Dirigir el cambio de la cultura organizacional hacia la calidad total. En: Cuadernos, 28. pp. 11-18.

- REAL ACADEMIA ESPAÑOLA. (2006). Diccionario Esencial de la Lengua Española. Madrid: Espasa Calpe.

- SERNA, H. (2008). Gerencia estratégica. Bogotá D.C.: 3R Editores.

- VESGA, J. J. (2011). Memorias del Seminario Gestión de la Cultura Organizacional. Cali: Editorial Bonaventuriana.

\section{Webgrafía}

- CONSEJO NACIONAL DE ACREDITACIÓN. (Enero de 2012). Lineamientos para la acreditación de programas de pregrado. Recuperado el 31 de Enero de 2013, desde: http://www.cna.gov.co/1741/articles-186359_pregrado_2012.pdf

$100<$ Universidad de San Buenaventura, Cali - Colombia 